\title{
MODELO DE APRENDIZAJE SOCIAL BASADO EN ANALITICAS: UN ENFOQUE PARA LA COLABORACIÓN CIENTIFICA
}

\section{MODEL SOCIAL LEARNING BASED ON ANALYTICS: A FOCUS FOR SCIENTIFIC COLLABORATION}

\author{
Daniel J. Salas ${ }^{1 *}$, Silvia M. Baldiris², Jorge E.Gómez ${ }^{3}$ \\ Recibido para publicación: 27 de abril 2017 - Aceptado para publicación: 8 de junio 2017
}

\begin{abstract}
RESUMEN
Este trabajo describe la estructura de un modelo de aprendizaje social basado en un enfoque analíticas para apoyar la adquisición de competencias científicas mediante procesos colaborativos, es decir, se busca definir el sistema de medición representado en analíticas, constructos, variables e indicadores que permitan evaluar la participación y/o aportes de los supervisores y el grado de relevancia de la participación de los investigadores aprendices en la construcción de un trabajo científico. Se ha definido un conjunto de fases que soportan el modelo constituido por tres partes fundamentales, la primera está asociada con el proceso de generación de analíticas, la segunda hace referencias a los actores para el proceso de elaboración de propuestas científicas y la tercera hace referencia al sistema de edición colaborativo y las herramientas de interacción social.
\end{abstract}

Palabras Clave: Aprendizaje social, Analíticas, Competencias Científicas, Colaboración, Variables, Indicadores

\begin{abstract}
ABSCTRACT
This paper describes the structure of a social learning model based on an analytical approach to support the acquisition of scientific competences through collaborative processes, that is, it seeks to define the measurement system represented in analytics, constructs, variables and indicators that allow evaluating the participation and / or contributions of the supervisors and the degree of relevance of the participation of the apprentice researchers in the construction of a scientific work. A set of phases has been defined that support the model constituted by three fundamental parts, the first is associated with the process of generating analytics, the second makes references to the actors for the process of preparing scientific proposals and the third makes reference to the collaborative editing system and social interaction tools.
\end{abstract}

Keyword: Social learning, Analytics, Scientific Competences, Collaboration, Variables, Indicators

\footnotetext{
1* M.Sc.en Informatica, Universidad Industrial de Santander UIS, Universidad de Córdoba, Montería, Colombia-email: dsalasalvarez@ correo.unicordoba.edu.co

${ }^{2}$ Ph. D. tecnologias de la informacion, Universidad de Girona. University of Thabasca.

${ }^{3}$ M.Sc. Telematica, Universidad de Córdoba, Departamento de Ingeniería de Sistemas, Montería - Córdoba
} 


\section{INTRODUCCIÓN}

Las Analíticas de Aprendizaje desde la perspectiva social[1], son una área potencial para apoyar de manera efectiva el aprendizaje, en este sentido, desde esta dimensión se prevé que los alumnos pueden interactuar y participar activamente mediante el desarrollo de actividades donde experimentan, comparten y colaboran mediante el uso de herramientas sociales que apoyados con técnicas de analíticas permiten dejar el registro de las acciones de los aprendices donde se identifican patrones y comportamientos de los estudiantes y soporte para el mejoramiento de su aprendizaje.

Los estudios de Buckinghan[2] y Fergunson[2] plantean cinco (5) categorías de las analíticas o de análisis desde la perspectiva social, ellas son: Análisis en Redes Sociales[3][4][5][6][7], Análisis del Discurso, Análisis del Contenido, Análisis de la disposición y Análisis del Contexto.

Dada las potencialidades para de las Analíticas de Aprendizaje en general, y en sus perspectivas social para medir y hacer eficiente los procesos de aprendizaje, en este trabajo de investigación, se intenta apoyar la adquisición de competencias científicas[8][9][10][11] de los jóvenes investigadores, específicamente en la elaboración de propuestas de investigación debido a las principales dificultades que enfrentan los mismos, cuando deben desarrollos procesos de investigación científicas, las dificultades más notorias están asociadas la poca disponibilidad de tutores para guiar a los jóvenes investigadores, la falta de claridad conceptual con respecto a los métodos y técnicas de investigación, dificultades para revisar la literatura científica, particularmente relacionada con la sistematización de la información recopilada, así como los problemas con la gestión de las fuentes bibliográficas. Sumado a todo esto, también la débil capacidad de establecer el diseño de la investigación, tanto con respecto a los enfoques cualitativos y cuantitativos, cuestiones intensificadas sobre el análisis e interpretación de los datos recopilados durante la investigación y los escenarios de evaluación, y sobre las condiciones y la forma en que deberían desarrollarse teniendo en cuenta los tipos de investigación y las situaciones de contexto.
Los estudios de Bazerman12[], Dudley-Evan[13] y Swales[14] han identificado que los principales problemas en la escritura científica, especialmente para los investigadores no nativos de países anglosajones, las dificultades más notorias son las siguientes: Dificultades en la gramática, uso de las citaciones, referencia a literatura publicada, estructuración de los argumentos, organización del texto.

- Relación del texto con la audiencia.

- Manera en la cual hacer afirmaciones de conocimiento.

- Manera de revelar u ocultar el punto de vista del autor

- Interferencia de diferentes puntos de vista culturales en la naturaleza del proceso académico.

Los estudios recientes de Sanchez Upegui[15]16[] también destacan la necesidad de establecer mecanismos y estrategias para la adecuada formulación de proyectos de investigación, por parte de los semilleros de investigación, jóvenes investigadores y estudiantes de postgrados, donde se observan dificultades en la formulación de la problemática, revisión de literatura, argumentación, estrategias de pensamiento critico y en temas asociados con la gramática.

Ahora bien, en nuestro contexto, los jóvenes de semilleros de investigación de la Universidad de Córdoba y en especial los 1,100 estudiantes del programa de Ingeniería de Sistemas, cuya metodología de formación es a distancia, donde los jóvenes se encuentran distribuidos en cuatro municipios del departamento (Montería, Lorica, Sahagún y Planeta Rica), también adolecen de las mismas problemáticas y dificultades para organizar propuestas de investigación, en lo relacionado con la formulación de problemas de investigación, la pregunta de investigación, dificultad para formular objetivos de investigación, dificultades para comprender los métodos de investigación, dificultad para interactuar y compartir experiencias entre estudiantes, dificultad para integrar docentes y estudiantes con la sedes, entre otros. Lo anterior, trae como consecuencias que las propuestas o trabajos de investigación tengan valoraciones muy por debajo de lo esperado, aunque muchos casos desde el punto de vista de la innovación contengan 
elementos significativos; también ocurre que los resultados alcanzados no permiten tener un mayor número de estudiantes con proyectos sobresalientes.

Los dificultades expuestas requieren nuevos enfoques que faciliten la colaboración, monitoreo y estrategias innovadoras relacionadas con los sistemas de medición que incorporan elementos que permiten reflexiones regulares sobre el proceso de formación en investigación junto con tutoría eficiente que incluye alertas tempranas en todo momento para los estudiantes, así como la facilitación de los mecanismos y los instrumentos que ayudan a alcanzar todas las competencias y habilidades que requiere el proceso de elaboración de propuestas de investigación. En este sentido, las Analíticas de Aprendizaje desde la perspectiva social, puede convertirse en una herramienta conceptual y tecnológica para apoyar eficazmente los procesos de formación científica, dado que permite conocer el comportamiento de los investigadores aprendices en todo el proceso formativo, conocer el estado actual de sus avances mediante el monitoreo permanente y además conocer el nivel de compromiso en el proceso de aprendizaje.

\section{METODOLOGÍA}

El proceso metodológico que se siguió para desarrollar el modelo de aprendizaje social tuvo en cuenta las siguientes fases: organización de la estructura del modelo, diseño e implementación de una red aprendizaje social para apoyar el desarrollo de competencias científicas, como marco general para colocar posteriormente en un escenario de prueba la validación del I modelo propuesto.

La segunda fase de este trabajo es el diseño e implementación de una red de aprendizaje social con enfoque colaborativo basado en analíticas para apoyar el desarrollo de competencias científicas de los investigadores aprendices a nivel de la escritura científica, es decir, que permite el desarrollo de habilidades en la formulación de propuestas científicas

\section{RESULTADOS}

\subsection{Elementos del Modelo Aprendizaje Social}

El modelo de aprendizaje social está conformado por tres elementos fundamentales, el primero está relacionado con la generación de analíticas asociadas con el diseño de propuestas de investigación, incluye además los sistemas de medición que incluyen las variables e indicadores, sistemas de recolección y análisis, mecanismo de visualización, monitoreo y avance del progreso de los investigadores, el segundo elemento está conformado por los principales actores del modelo que son los revisores o supervisores y los investigadores aprendices, los primeros se encargan de dirigir, asesorar, apoyar y acompañar a los investigadores aprendices en la formulación de propuestas de investigación científicas, los investigadores aprendices son los autores de las propuestas que buscan adquirir las competencias y/o habilidades en la redacción científica.

El tercer elemento del modelo lo conforman cuatro aspectos fundamentales: Sistema de Colaboración para la edición de propuestas científicas, interacción social para el aprendizaje, proceso de auto reflexión para el análisis de comportamientos y resultados de procesos apropiación.

El sistema de edición colaborativa busca que los investigadores aprendices construyan sus propuestas de investigación participando activamente con sus supervisores, atendiendo los comentarios y/o observaciones en cada uno de los elementos que conforman la estructura del proyecto de investigación que se pretende formular, por otra parte el proceso interacción social incluye la participación herramientas de discusión, seguir a supervisores o investigadores en áreas de interés, chat para discutir aspectos significativos de los componentes de las propuestas de investigación, auto reflexiones a partir los comentarios de las participaciones en la construcción de las propuestas de investigación y finalmente los resultados alcanzados en el proceso de construcción de los trabajos científicos. 
Figura 1. Modelo de aprendizaje social.

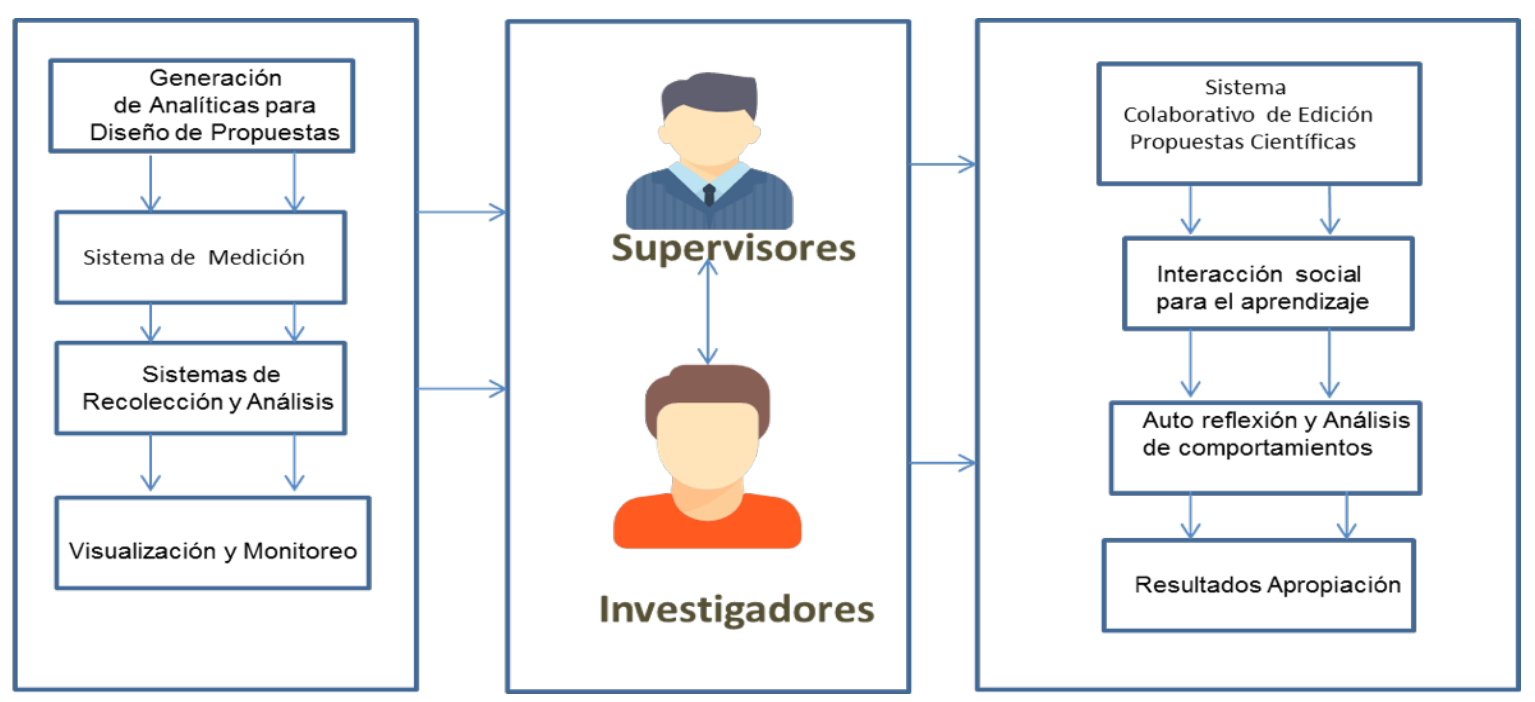

\subsection{Descripción del Modelo de Aprendizaje social}

\subsubsection{Generación de Analíticas para el diseño de propuestas de investigación}

En esta sección se definen las Analíticas de Aprendizaje relativas al proceso de formulación de una propuesta de investigación, es decir, se intenta establecer a grandes rasgos el objeto de análisis que se pretende explorar y sus respectivas dimensiones, en este sentido, se han definido seis (6) analíticas que comprenden el proceso de investigación científica.

A1 - Relativas a la calidad en el proceso de formulación de problemas y preguntas de investigación

A2 - Concerniente a la calidad de la revisión de literatura y la apropiación conceptual y práctica de los investigadores en formación.

A3 - Inherentes a las capacidades de los estudiantes en la definición de los objetivos,, justificación y en el enfoque y alcance de la investigación y la calidad de los mismos

A4 - Relacionados con las capacidades de los investigadores en formación para plantear calidad en el diseño de investigación.

A5 - Relativas a la calidad en la recolección y en el análisis de datos por parte de los investigadores principiantes

A6 - Relativas a la calidad en la interpretación de datos y los informes científicos que realizan los investigadores principiantes.

\subsubsection{Sistemas de Medición}

Los sistemas de medición comprenden las variables o constructos que se desean medir, igual que instrumentos e indicadores que se utilizan para tal fin, teniendo en cuenta la confiabilidad de los mismos con el propósito de minimizar los errores en el proceso.

Dado que se quiere medir las habilidades de los investigadores aprendices o principiantes el proceso de formulación de propuestas de investigación científica se definen constructos o variables en la siguiente sección

En esta sección se definen 14 constructos o variables que se desean medir en el proceso de formulación de propuestas científicas de los investigadores en formación con el fin de conocer las capacidades que obtendrán dichos investigadores y determinar cómo ha sido el proceso de apropiación de los conceptos y desarrollo de las habilidades de cada uno de los elementos o componentes que integran el proceso investigativo. 
Tabla 1. Constructos y sistema de medidas

\begin{tabular}{|c|c|c|}
\hline Variable (Constructos) & Definición & Medida \\
\hline \multirow{3}{*}{$\begin{array}{l}\text { H1: Habilidad para plantear el problema } \\
\text { de investigación a partir de un conjunto de } \\
\text { observaciones, datos y referentes teóricos. }\end{array}$} & \multirow{3}{*}{$\begin{array}{l}\text { Conocimiento y capacidades de los investiga- } \\
\text { dores aprendices para formular problemas de } \\
\text { investigación }\end{array}$} & $\begin{array}{l}\text { Cuestionario pretest } \\
\text { (Confiabilidad Cronbach) }\end{array}$ \\
\hline & & Indicador de Colaboración \\
\hline & & Rubrica \\
\hline \multirow[t]{3}{*}{$\begin{array}{l}\text { H2 : Habilidad para construir preguntas } \\
\text { de investigación }\end{array}$} & \multirow[t]{3}{*}{$\begin{array}{l}\text { Definir y estructurar preguntas de investiga- } \\
\text { ción científica }\end{array}$} & $\begin{array}{l}\text { Cuestionario pretest } \\
\text { Confiabilidad Conbranch ) }\end{array}$ \\
\hline & & Indicador de Colaboración \\
\hline & & Rubrica \\
\hline \multirow{4}{*}{$\begin{array}{l}\text { H3:. Habilidad para estructurar metodológi- } \\
\text { camente un proceso de revisión de literatura } \\
\text { partiendo de preguntas de investigación } \\
\text { concretas }\end{array}$} & \multirow[t]{4}{*}{$\begin{array}{l}\text { Describir y estructurar el proceso para una } \\
\text { revisión sistemática }\end{array}$} & $\begin{array}{l}\text { Cuestionario pretest } \\
\text { (Confiabilidad Conbranch ) }\end{array}$ \\
\hline & & Indicador de Colaboración \\
\hline & & Rubrica \\
\hline & & Rubrica \\
\hline \multirow[t]{3}{*}{$\begin{array}{l}\text { H5: Habilidad para sintetizar los datos prove- } \\
\text { nientes de una literatura en particular. }\end{array}$} & \multirow[t]{3}{*}{$\begin{array}{l}\text { Capacidad para categorizar, clasificar y agru- } \\
\text { par información de una revisión de literatura. }\end{array}$} & $\begin{array}{l}\text { Cuestionario pretest } \\
\text { (Confiabilidad Conbranch ) }\end{array}$ \\
\hline & & Indicador de Colaboración \\
\hline & & Rubrica \\
\hline \multirow[t]{3}{*}{$\begin{array}{l}\text { H6: Habilidad para reportar los hallazgos en } \\
\text { una revisión de literatura. }\end{array}$} & \multirow[t]{3}{*}{$\begin{array}{l}\text { Capacidad para visualizar los hallazgos en } \\
\text { una revisión literaria. }\end{array}$} & $\begin{array}{l}\text { Cuestionario pretest } \\
\text { (Confiabilidad Conbranch ) }\end{array}$ \\
\hline & & Indicador de Colaboración \\
\hline & & Rubrica \\
\hline \multirow[t]{3}{*}{$\begin{array}{l}\text { H7: Habilidad para construir correctamente } \\
\text { los objetivos de investigación }\end{array}$} & \multirow[t]{3}{*}{$\begin{array}{l}\text { Formular apropiadamente objetivos de inves- } \\
\text { tigación }\end{array}$} & $\begin{array}{l}\text { Cuestionario pretest } \\
\text { (Confiabilidad Conbranch ) }\end{array}$ \\
\hline & & Indicador de Colaboración \\
\hline & & Rubrica \\
\hline \multirow[t]{3}{*}{$\begin{array}{l}\text { H8: Habilidad para justificar teórica y práctica- } \\
\text { mente la investigación }\end{array}$} & \multirow[t]{3}{*}{$\begin{array}{l}\text { Facilidad para argumentar la importancia y } \\
\text { relevancia de una investigación }\end{array}$} & $\begin{array}{l}\text { Cuestionario pretest } \\
\text { (Confiabilidad Conbranch ) }\end{array}$ \\
\hline & & Indicador de Colaboración \\
\hline & & Rubrica \\
\hline \multirow[t]{3}{*}{$\begin{array}{l}\text { H9: Habilidad para definir el enfoque y el } \\
\text { alcance de la investigación }\end{array}$} & \multirow[t]{3}{*}{$\begin{array}{l}\text { Capacidad para definir las limitaciones de la } \\
\text { investigación }\end{array}$} & $\begin{array}{l}\text { Cuestionario pretest } \\
\text { (Confiabilidad Conbranch ) }\end{array}$ \\
\hline & & Indicador de Colaboración \\
\hline & & Rubrica \\
\hline \multirow{3}{*}{$\begin{array}{l}\text { H10: Habilidad para plantear diseño de investi- } \\
\text { gación apropiado de acuerdo con los objetivos, } \\
\text { el enfoque y el alcance de la investigación. }\end{array}$} & \multirow[t]{3}{*}{$\begin{array}{l}\text { Manejo conceptual para aplicar el diseño a } \\
\text { un proceso de investigación científica }\end{array}$} & $\begin{array}{l}\text { Cuestionario pretest } \\
\text { (Confiabilidad Conbranch ) }\end{array}$ \\
\hline & & Indicador de Colaboración \\
\hline & & Rubrica \\
\hline \multirow{3}{*}{$\begin{array}{l}\text { H11: Habilidad para utilizar procedimientos } \\
\text { apropiados de recolección de datos conside- } \\
\text { rando variables contextuales }\end{array}$} & \multirow[t]{3}{*}{$\begin{array}{l}\text { Capacidad para identificar procedimientos e } \\
\text { instrumentos para la recolección de datos }\end{array}$} & $\begin{array}{l}\text { Cuestionario pretest } \\
\text { (Confiabilidad Conbranch ) }\end{array}$ \\
\hline & & Indicador de Colaboración \\
\hline & & Rubrica \\
\hline
\end{tabular}




\begin{tabular}{|c|c|c|}
\hline Variable (Constructos) & Definición & Medida \\
\hline \multirow[t]{3}{*}{$\begin{array}{l}\text { H12 : Habilidad para la creación de instrumen- } \\
\text { tos de recolección de datos }\end{array}$} & \multirow[t]{3}{*}{$\begin{array}{l}\text { Capacidad definir, describir y utilizar instru- } \\
\text { mentos de recolección de datos. }\end{array}$} & $\begin{array}{l}\text { Cuestionario pretest } \\
\text { (Confiabilidad Cronbanch ) }\end{array}$ \\
\hline & & Indicador de Colaboración \\
\hline & & Rubrica \\
\hline \multirow[t]{3}{*}{$\begin{array}{l}\text { H13: Habilidad para la identificación de las } \\
\text { técnicas más adecuada de análisis de datos. }\end{array}$} & \multirow[t]{3}{*}{$\begin{array}{l}\text { Capacidad describir y utilizar las técnicas y } \\
\text { procedimientos de análisis de datos }\end{array}$} & $\begin{array}{l}\text { Cuestionario pretest } \\
\text { (Confiabilidad Conbranch ) }\end{array}$ \\
\hline & & Indicador de Colaboración \\
\hline & & Rubrica \\
\hline \multirow{3}{*}{$\begin{array}{l}\text { H14: Habilidad en el manejo de herramientas } \\
\text { tecnológicas para el análisis de datos en una } \\
\text { investigación }\end{array}$} & \multirow[t]{3}{*}{$\begin{array}{l}\text { Conocimiento y comprensión de las herra- } \\
\text { mientas para el análisis de datos }\end{array}$} & $\begin{array}{l}\text { Cuestionario pretest } \\
\text { (Confiabilidad Conbranch ) }\end{array}$ \\
\hline & & Indicador de Colaboración \\
\hline & & Rubrica \\
\hline
\end{tabular}

\subsubsection{Cuestionario Pre test}

Este cuestionario busca conocer los conocimientos previos de los investigadores aprendices en relación con la formulación de propuesta de investigación, es decir, si tienen habilidades en la formulación de preguntas de investigación, planteamientos de problemas, hipótesis, revisión de literatura, formulación de objetivos, justificación de una investigación, definir y establecer el diseño metodológico, establecer las contribuciones esperadas de la investigación y sustentar las referencias que soportan el trabajo.

\subsubsection{Ponderación de Componentes}

La ponderación de componentes es una medida que determina el peso o relevancia de cada uno de elementos que conforman una propuesta de investigación, que varía de acuerdo a los expertos y a las instituciones, para el caso de la Universidad de Córdoba, se tiene la siguiente ponderación.

Tabla 2. Ponderación de Componentes

\begin{tabular}{|c|c|}
\hline Componentes propuesta investigación & Ponderación. \\
\hline Planteamiento del Problema & $10 \%$ \\
\hline Pregunta de Investigación & $10 \%$ \\
\hline Justificación & $10 \%$ \\
\hline Objetivos & $10 \%$ \\
\hline Alcances & $5 \%$ \\
\hline
\end{tabular}

\begin{tabular}{|c|c|}
\hline Revisión de Literatura/Estado del Arte & $15 \%$ \\
\hline Metodología & $10 \%$ \\
\hline Cronograma de Actividades & $5 \%$ \\
\hline Resultados Esperados & $5 \%$ \\
\hline Presupuesto & $5 \%$ \\
\hline Referencias & $5 \%$ \\
\hline Total & $100 \%$ \\
\hline
\end{tabular}

\subsubsection{Indicadores de Revisión colaborativa de Supervisores}

Los indicadores de revisión reflejan los aportes, sugerencias o recomendaciones que los supervisores realizan para mejorar las propuestas de los investigadores aprendices.

Las notaciones utilizadas en la tabla 3 expresan lo siguiente:

Componente: Hace parte de cada ítems de las cuales está conformado una propuesta o proyecto de investigación.

Ponderación: Es el grado de importancia - relevancia que tiene cada ítems de los componentes de una propuesta de investigación y varía de acuerdo a la institución o tipo de formación que se quiera evaluar.

Revisor: Son los expertos encargados de evaluar o supervisar las propuestas de investigación que 
elaboran los autores.

$\mathrm{N}_{1}=$ Numero de comentarios del revisor uno (1)

$\mathrm{N}_{2}=$ Numero de comentarios del revisor dos (2)
\%PR1 = Porcentaje de participación del revisor uno (1)

\%PR2 = Porcentaje de participación del revisor dos (2)

Tabla 3. Indicadores de revisión.

\begin{tabular}{|c|c|c|c|c|c|}
\hline $\begin{array}{c}\text { Componentes propuesta } \\
\text { investigación }\end{array}$ & Ponderación. & $\begin{array}{c}\text { Revisor1 } \\
\text { (Comentarios) }\end{array}$ & $\begin{array}{c}\text { Revisor } 2 \\
\text { (Comentarios) }\end{array}$ & \%PR1 & \%PR2 \\
\hline Planteamiento del Problema & $10 \%$ & $N_{1}$ & $\mathrm{~N}_{2}$ & $\% P R 1=N 1 * 10 / N 1+N 2$ & $\% P R 2=N 2 * 10 / N 1+N 2$ \\
\hline Pregunta de Investigación & $10 \%$ & $\mathrm{~N}_{1}$ & $\mathrm{~N}_{2}$ & $\% P R 1=N 1 * 10 / N 1+N 2$ & $\% P R 2=N 2 * 10 / N 1+N 2$ \\
\hline Justificación & $10 \%$ & $\mathrm{~N}_{1}$ & $\mathrm{~N}_{2}$ & $\% P R 1=N 1 * 10 / N 1+N 2$ & $\% P R 2=N 2 * 10 / N 1+N 2$ \\
\hline Objetivos & $10 \%$ & $\mathrm{~N}_{1}$ & $\mathrm{~N}_{2}$ & $\% P R 1=N 1 * 10 / N 1+N 2$ & $\% P R 2=N 2 * 10 / N 1+N 2$ \\
\hline Alcances & $5 \%$ & $\mathrm{~N}_{1}$ & $\mathrm{~N}_{2}$ & $\% P R 1=N 1 * 5 / N 1+N 2$ & $\% P R 2=N 2 * 5 / N 1+N 2$ \\
\hline $\begin{array}{c}\text { Revisión de Literatura/Estado } \\
\text { del Arte }\end{array}$ & $15 \%$ & $\mathrm{~N}_{1}$ & $\mathrm{~N}_{2}$ & $\% P R 1=N 1 * 15 / N 1+N 2$ & $\% P R 2=N 2 * 15 / N 1+N 2$ \\
\hline Metodología & $10 \%$ & $\mathrm{~N}_{1}$ & $\mathrm{~N}_{2}$ & $\% P R 1=N 1 * 10 / N 1+N 2$ & $\% P R 2=N 2 * 10 / N 1+N 2$ \\
\hline Cronograma de Actividades & $5 \%$ & $\mathrm{~N}_{1}$ & $\mathrm{~N}_{2}$ & $\% P R 1=N 1 * 5 / N 1+N 2$ & $\% P R 2=N 2 * 5 / N 1+N 2$ \\
\hline Resultados Esperados & $5 \%$ & $\mathrm{~N}_{1}$ & $\mathrm{~N}_{2}$ & $\% P R 1=N 1 * 5 / N 1+N 2$ & $\% P R 2=N 2 * 5 / N 1+N 2$ \\
\hline Presupuesto & $5 \%$ & $\mathrm{~N}_{1}$ & $\mathrm{~N}_{2}$ & $\% P R 1=N 1 * 5 / N 1+N 2$ & $\% P R 2=N 2 * 5 / N 1+N 2$ \\
\hline Referencias & $5 \%$ & $\mathrm{~N}_{1}$ & $\mathrm{~N}_{2}$ & $\% P R 1=N 1 * 5 / N 1+N 2$ & $\% P R 2=N 2 * 5 / N 1+N 2$ \\
\hline
\end{tabular}

\subsubsection{Indicadores de Participación Colaborativa de los Investigadores Aprendices}

Este indicador muestra el compromiso de los investigadores en el proceso de construcción de la propuesta de investigación y tiene básicamente dos componentes, la edición colaborativa del proyecto de investigación y la interacción y participación en las herramientas sociales, tales como foros, chat y compartición de documentos, entre otros.

Las notaciones para los indicadores de participación de los investigadores se relacionan a continuación:

Investigadores: Autores de las propuestas de investigación

Supervisores: Responsables de revisar las propuestas de investigación

PAS: Porcentaje de acceso al sistema.

PEPPIN: Porcentaje de edición de propuestas de investigación
PMPI: Porcentaje de modificación de propuestas de investigación

PRCS: Porcentaje de respuesta a los comentarios de los supervisores

PPF: Porcentaje de post participación en foros.

\%Acceso: Porcentaje de acceso de los investigadores al sistema

Ntotall1: Número total de accesos del investigador 1

Notalacesso: Número total de accesos al sistema NEl1: Numero de ediciones del investigador uno(1), dos( 2), tres(3), (n)

Ntediciones: Número total de ediciones

Nmpipi1: Numero de modificaciones por ítems de la propuesta de investigación del investigador uno(1), dos(2), tres (3), (n)

Ntotalmp= Número total de modificaciones por ítems de la propuesta de investigación.

Ntri1: Número total de respuesta del investigador uno(1), dos(2), tres(3)

Ntpl1: Número total de post del investigador uno(1), dos(2), tres(3)

Ntotalp: Número total de post en el foro. 


\begin{tabular}{|c|c|c|c|c|c|}
\hline Investigadores & PAS & PEPPIN & PMPI & PRCS & PPF \\
\hline 1 & $\%$ Acceso $=100 * \frac{\text { NoTal } 1}{\text { Ntotalacceso }}$ & $\%$ PEPPIN $=100 * \frac{\text { NEI } 1}{\text { Ntediciones }}$ & $\begin{array}{l}\% P M P I \\
=100 \\
* \text { NMPIPI1/Ntotalmp }\end{array}$ & $\begin{array}{l}\text { \%PRCS } \\
=100 \\
* \text { Ntrl1/Ntoalresp }\end{array}$ & $\begin{array}{l}\% P P F \\
=100 * \text { NtpI } 1 / \text { Ntotalp }\end{array}$ \\
\hline 2 & $\%$ Acceso $=100 * \frac{\text { NoTal } 2}{\text { Ntotalacceso }}$ & $\%$ PEPPIN $=100 * \frac{N E I 2}{\text { Ntediciones }}$ & $\begin{array}{l}\% P M P I \\
=100 \\
* N M P I P I 2 / \text { Ntotalmp }\end{array}$ & $\begin{array}{l}\text { PRCS } \\
=100 \\
* \text { NtrI2/Ntoalresp }\end{array}$ & $\begin{array}{l}\% P P F \\
=100 * \text { NtpI } / \text { Ntotalp }\end{array}$ \\
\hline 3 & $\%$ Acceso $=100 * \frac{\text { NoTal } 3}{\text { Ntotalacceso }}$ & $\%$ PEPPIN $=100 * \frac{\text { NEI3 }}{\text { Ntediciones }}$ & $\begin{array}{l}\% P M P I \\
=100 \\
* \text { NMPIPI3/Ntotalmp }\end{array}$ & $\begin{array}{l}\text { PRCS } \\
=100 \\
* \text { Ntrl3/Ntoalresp }\end{array}$ & $\begin{array}{l}\% P P F \\
=100 * \text { NtpI3/Ntotalp }\end{array}$ \\
\hline $\mathrm{N}$ & $\%$ Acceso $=100 * \frac{\text { NoTalN }}{\text { Ntotalacceso }}$ & $\%$ PEPPIN $=100 * \frac{N E 1 N}{\text { Ntediciones }}$ & $\begin{array}{l}\% P M P I \\
=100 \\
* \text { NMPIPIN } / \text { Ntotalmp }\end{array}$ & $\begin{array}{l}\text { PRCS } \\
=100 \\
* \text { NtrIn/Ntoalresp }\end{array}$ & $\begin{array}{l}\% P P F \\
=100 * \text { Ntpl3/Ntotalp }\end{array}$ \\
\hline
\end{tabular}

Tabla 4. Indicadores de participación de los investigadores

\subsubsection{Rubricas para evaluación de competencias}

Se han definido tiene siete (7) rubricas con 21 criterios de evaluación que dan cuenta de la verificación de las competencias relacionadas con el proceso investigativo, de tal manera que permita que cada revisor o supervisor pueda evaluar el progreso de cada investigador principiante teniendo en cuenta dichos criterios

\subsection{Sistemas de recolección y análisis}

La recolección de datos se realiza a través de la aplicación de los instrumentos, tales como cuestionarios y rubricas, por otra parte se almacenará la información derivada de la interacción de los actores( investigadores y supervisores) en el editor colaborativo en el proceso continuo de construcción de las propuesta de investigación, además se recolecta en los Logs de sistema y la información de la interacción en las herramientas sociales tales como foros, chat, sistemas para compartir documentos y seguimiento de usuarios según su perfil.

Las herramientas para el análisis de la información son a través de algoritmos de agrupamientos y clasificación y análisis de comportamientos individuales teniendo en cuenta la información generada con el procesamiento de los de los indicadores.

\subsection{Visualización y Monitoreo de propuestas}

Los mecanismos de visualización muestran las formas de progreso de los investigadores en el proceso de construcción de las propuestas de investigación, indicando en términos de tiempos los avances o tareas pendientes de acuerdo con el tiempo establecido para el desarrollo del proyecto.

\subsection{Actores}

Los principales actores de este modelo son los revisores o supervisores y los investigadores principiantes, los primeros son los expertos en encargados de evaluar y verificar la calidad de los avances de los proyectos o propuestas de investigación, mientras que los investigadores aprendices asumen el proceso colaborativo de construcción de los trabajos de investigación en los tiempos previstos y asumiendo las consideraciones derivadas de las revisiones de los revisores.

\section{Sistemas Colaborativo de Edición de Propuestas Científicas}

Este sistema permite que tanto los investigadores y los supervisores puedan trabajar colaborativamente en el proceso de construcción o elaboración de las propuestas de investigación, llevando registro de todas las acciones asociadas con la educación y/o modificación de las propuestas que además está articulado con el 
sistema de notificaciones.

\subsection{Interacción social para el aprendizaje}

Se realiza a través del acceso a herramientas de comunicación, tales como foros de discusión, compartición de documentos, seguir a usuarios con intereses comunes, acceso a chat, entre otros, permite que los investigadores y revisores puedan realizar interacciones para discutir asunto acerca del proceso de construcción de las propuestas de investigación

\subsection{Auto reflexión}

Hace referencia al estado de avance de los investigadores aprendices de sus propuestas de investigación en cada uno de sus componentes, especificando los detalles del proceso para que con base en ello, reflexione y evalué la ruta que puede seguir para alcanzar las competencias requeridas

\subsection{Resultados de Apropiación}

Indica los logros o competencias alcanzadas por los investigadores en el proceso de construcción de las propuestas de investigación en el marco de los tiempos establecidos, siguiendo las orientaciones del revisor o supervisores del trabajo de investigación.

\section{CONCLUSIONES}

En este trabajo se presentó un modelo de aprendizaje social basado en analíticas que describe las fases, sistemas de medidas, actores y herramientas sociales para apoyar la adquisición de competencias científicas a niveles de elaboración de propuestas de investigación, esta representación del modelo constituye la primera fase para la implementación del mismo mediante la disposición de una plataforma tecnológica que permita validar en un escenario formativo la relevancia de la propuesta en mención.

\section{REFERENCIAS}

[1.] Fergunson, R. et al (2012) Social Learning Analytics: Five Approaches

[2.] Buckingham,S, et al (2012) Social Learning Analytics, Educational Technology \& Society, 15 (3), 3-26.

[3.] Dawson S. (2008). A study of the relationship between student social networks and sense of community. Educational Technology \& Society, 11(3), 224-238

[4.] Dawson, S., et al (2010). SNAPP: Realising the affordances of real-time SNA within networked learning environments. Networked Learning - Seventh International Conference, Aalborg, Denmark.

[5.] Dawson, S., et al (2011) Using Social Network Metrics to Assess the Effectiveness of Broad-Based Admission Practices. Australasian Journal of Educational Technology, 27(1), 16-27.

[6.] Dawson S. (2008). A study of the relationship between student social networks and sense of community. Educational Technology \& Society, 11(3), 224-238

[7.] Blackmore, C. (Ed.). (2010). Social learning systems and communities of practice. London, the United Kingdom: Springer

[8.] UNESCO,(2005) ¿ Cómo promover el interés por la cultura científica? Una propuesta didáctica fundamentada para la educación científica de jóvenes de 15 a 18 años

[9.] OCDE, PISA,(2006) Marco de la evaluación. Conocimiento y habilidades en ciencias, matemáticas y lectura.

[10.] Hernández, C, (2005) Qué son las competencias científicas, Foro Educativo Nacional.

[11.] Jaramillo, H,(2006). Formación en habilidades y competencias científicas con base en la nano ciencias y la nanotecnología en la básica secundaria y media. 
[12.] Bazerman, Ch. (1988) Shaping Written Knowledge: The genre and activity of the experimental article in sciencie, Wisconsin : university of Wisconsin Press.

[13.] Dudley- evans, T. (1994) Genre Analysis: an approach text analysis for ESP. En Coulthard(ed) Advances in written text analysis (pp 219-228)

[14.] Swales, J. (1981) Aspect of articles introductions Birmingham: University osf Aston
[15.] Sánchez, A, (2016) El género articulo científico: escritura y análisis desde la alfabetización académica y la retórica funcional, Fundación Universitaria Católica del Norte, ISSN 978- 958- 8776- 50-7.

[16.] Sánchez, A, (2012). Revisión sobre el análisis lingüístico de artículos científicos: Una estrategia de alfabetización académica de orden superior, Fundación Universitaria Católica del Norte 